\title{
Endogenous Growth with Intertemporally Dependent Preferences*
}

\author{
Giuseppe Ferraguto \\ Istituto di Economia Politica \\ Bocconi University
}

\author{
Patrizio Pagano \\ Research Department \\ Bank of Italy
}

March 28, 2003

\begin{abstract}
This paper presents an endogenous growth model with intertemporally dependent preferences and " $\mathrm{Ak}$ " technology. We derive sufficient conditions for a balanced growth path to be an equilibrium, provide a full characterization of the equilibrium dynamics of the economy, and explore the implications of habit formation for the evolution of wealth distribution. Our results highlight the importance of preferences in the dynamics of growth, a point neglected in most of the literature.
\end{abstract}

Keywords: Preferences, Habits, Growth.

JEL classification numbers: D91, O41, E32.

${ }^{*}$ We thank, without implicating Chad Jones and Guido Tabellini, for helpful suggestions. Errors are our own. The opinions expressed in this paper do not necessarily reflect those of the Bank of Italy. Ferraguto: Via U. Gobbi, 5 - 20136 Milan, Italy; email: giuseppe.ferraguto@uni-bocconi.it, phone: (+39)-02-5836.5408; Pagano: Via Nazionale 91 - 00184 Rome, Italy, e-mail: pagano.patrizio@insedia.interbusiness .it, phone: (+39)-06-4792.3326. 


\section{Introduction}

This paper presents an endogenous growth model that departs from the assumption of time-separable, constant intertemporal elasticity of substitution (CIES) preferences almost ubiquitous in the literature. The idea, shared with Ryder and Heal (1973), is rather that it is intuitively plausible to assume that past consumption choices and/or the social environment affect the utility an individual derives from consuming a given bundle of goods. Therefore, we assume that the representative agent's instantaneous utility $u$ is determined by comparing current consumption $c$ to some reference stock, or standard, $z$, called alternatively "consumption experience", "habits", or "customary consumption", so that $u(c, z)$. With $z$ taken to be a weighted average of past consumption levels, this choice leads to preferences that Ryder and Heal termed "intertemporally dependent".

These preferences represent a tractable departure from the hypothesis of a time-separable utility function almost ubiquitous in the literature, and have been used in a variety of different contexts. To mention just a few, Ryder and Heal $(1973)$ and Boyer $(1975,1978)$ investigated their implications for the neoclassical optimal growth model, showing that they lead to a richer dynamic behavior of the main variables around an unchanged steady-state (the modified golden rule). Time non-separable preferences can help to reconcile rational choice theory with apparently irrational behavior (Becker and Murphy, 1983), to explain various time-series features of consumption data (Deaton, 1992), and to shed light on open economy macroeconomic issues (Obstfeld, 1992; Mansoorian, 1993). Finally, time nonseparable preferences have more recently been used in finance, often in the attempt to resolve the "equity premium puzzle" (Constantinides (1990), Abel (1990), Gali (1994), Boldrin et al. (1997), Campbell and Cochrane (1999)), and in business cycle research (Lattau and Uhlig (2000), Ljungqvist and Uhlig (2000), Otrok (2001)).

This paper is closely related to Carroll, Overland and Weil $(1997,2000)$ and the results we derive complement those obtained in these papers. The first (Carroll et al., 1997) analyzes the implications of different assumptions about how the representative agent's stock of habits evolves. It shows that both under "external habits" — where the stock of habits is a weighted average of the aggregate per-capita past consumption levels - and under "internal habits" - where an individual's habits are accumulated by his own consumption - the steady state growth rate of the economy is the same and, qualitatively, also the transitional dynamics to the balanced growth path. The companion paper (Carroll et al., 2000) shows the important implications 
of habit forming preferences for understanding the growth to saving causality apparent in the data.

As in these papers, we introduce the assumption of intertemporally dependent preferences in an otherwise standard $A k$ growth model, but the analysis that follows differs from theirs both in emphasis and generality.

In fact, rather than assuming from the outset a specific functional form for the instantaneous utility function, as these authors do, we work with a generic $u$, and provide sufficient conditions that this latter has to meet for a balanced growth path to qualify as an equilibrium when preferences are intertemporally dependent. This also allows us to unveil the difference between the "adjacent" and "distant" complementarity cases, and the ensuing dynamics, which is central to most of the literature on habit formation. Under adjacent complementarity, an increase in consumption experience induces the individual to want to increase current consumption, so that $c$ and $z$ will be positively related in equilibrium. The opposite is true when preferences are such that complementarity is distant. We choose to focus mainly on the case of adjacent complementarity, as we regard the addictive behavior it implies as more relevant in the one-sector framework we consider, where $c$ has to be interpreted as consumption of a wide bundle of goods. ${ }^{1}$ Nevertheless, our analysis also encompasses the opposite case of complementarity, and all the results we present can be readily extended to consider the implications of this alternative behavioral assumption.

Second, rather than transforming the problem in terms of state-like and control-like variables, we study the problem by introducing detrended variables, as in Caballé and Santos (1993), and by linearizing the dynamic system around the steady state. This allows us to obtain simple explicit solutions for the implied time paths of the main variables. Furthermore, we adapt and extend a graphical device first introduced by Obstfeld (1992) in his analysis of a small open economy facing a constant world interest rate, to provide a pictorial representation of the equilibrium dynamics resulting from our growth model with intertemporally dependent preferences. This representation is, we believe, both simple and transparent, and helps to grasp in an intuitive way the somewhat tangled interactions among variables that set in under habit formation.

Finally, having provided a full characterization of the equilibrium dynamics under "inward" - or "internal" - habit formation, the explict solu-

\footnotetext{
${ }^{1}$ Besides being theoretically plausible, this case seems also to be empirically relevant, as Fuhrer and Klein (1998) - who provide evidence suggesting that habit formation characterizes aggregate consumption behavior among most of the G-7 countries by testing a model that implies adjacent complementarity - have recently shown.
} 
tion for the evolution of the level of the main variables, which will depend on initial habits and capital stock, allows us to assume heterogeneity in the initial conditions and focus on the implications for the evolution of the distribution of capital. In fact, we show that intertemporally dependent preferences can generate a variety of outcomes in terms of time evolution of the ratio of individual capital to the mean or average capital in the economy: they include divergence, convergence, and leapfrogging. The model predicts that along the transition towards the steady state, individuals that start off with an initial endowment of capital to habits ratio which is high relative to the average one will increase their distance from the mean pushing towards more inequality. On the other hand, those individuals characterized by a relatively low capital to habits ratio should display consumption and capital holdings converging towards the mean. Which of the two effects will eventually prevail is therefore a matter of the initial distributions of capital and habits. Furthermore, a permanent increase in the equilibrium rate of growth, following say a productivity shock, can either generate increasing or decreasing inequality according to the distribution of initial conditions. These effects are absent in the standard time separable case (Rebelo, 1991), where there is no transitional dynamics and the distribution is always the same, and, as we will show below, are more rich than those stemming from the assumption of a Stone-Geary instantaneous utility function, a different departure from the hypothesis of CIES preferences.

The rest of the paper is organized as follows. Section 2 presents the model. Section 3 introduces the concepts of equilibrium adopted and gives sufficient conditions for the existence of a steady-state balanced growth path. In Section 4, through a normalization of variables, we transform our original problem into one that involves only variables that take on constant values in balanced growth, and provide a full characterization of the equilibrium dynamics of the latter. In Section 5 we present a graphical device that helps determine the equilibrium dynamics of the economy starting from an arbitrary set of initial conditions on the state variables, and provide the economic intuition for the transitional dynamics implied by intertemporally dependent preferences. In Section 6 we present an example of instantaneous utility function belonging to the intertemporally dependent class and discuss the implications of our model in terms of the evolution of the cross-section of capital holdings. Section 7 concludes. 


\section{The model}

We study a closed economy with an unbounded horizon, populated by infinitely lived, identical individuals. The representative agent has preferences defined over his own consumption $c$, as well as on consumption experience, or habits, $z$, and maximizes the objective functional:

$$
U(c, z)=\int_{0}^{\infty} e^{-\delta t} u(c(t), z(t)) d t
$$

where $u$ is the instantaneous felicity function, and $\delta$ the (positive and constant) rate of time preference.

As in Ryder and Heal (1973), we assume that consumption experience is a weighted average of the representative individual's past consumption levels $c$,

$$
z(t)=z_{0} e^{-\rho t}+\rho \int_{0}^{t} e^{-\rho(t-s)} c(s) d s,
$$

where $\rho>0$ is a constant that measures the rate of habit adjustment, and $z(0)=z_{0}>0$ is the exogenously inherited standard of living at the initial date. The larger is $\rho$, the higher the weight given to past consumption in determining the current level of consumption experience, and vice versa. ${ }^{2}$

Differentiating equation (2) with respect to time, it follows that habits evolve according to

$$
\dot{z}(t)=\rho[c(t)-z(t)]
$$

We impose the following conditions on the instantaneous felicity function $u$, assumed to be twice continuously differentiable:

\footnotetext{
${ }^{2}$ A substantial modification would stem from the assumption that the representative agent's stock of habits is a weighted average of the aggregate per-capita (or average, or the Joneses') past consumption levels $\chi$, rather than of his own past consumption $c$. The implications of this alternative assumption are studied by Carroll et al. (1997). Since the representative agent takes $\chi$ as given, although $\chi=c$ must hold in equilibrium, it introduces a "consumption externality" that breaks the equivalence between the centralized and competitive solutions of the model, with potential policy implications which are absent when habits are as in (2). Aside from this, the assumption that habits are accumulated by $c, \chi$, or by a weighted average of the two does not change the steady state growth rate of the economy, and does not lead to qualitatively different transitional dynamics to the balanced growth path that are the main object of the present paper.
} 
U1. $u_{c}>0 ; \lim _{c \rightarrow 0} u_{c}=\infty, \quad z \in(0, \infty)$;

U2. $u_{z} \neq 0 ; u_{c z} \neq 0$;

U3. $u_{c c}<0, \quad u_{c c} u_{z z}-u_{c z}^{2} \geq 0$

U4. $u_{c}+\left(\frac{\rho}{\rho+g}\right) u_{z}>0 \quad \forall g>0$.

Assumption U2 restricts preferences to the intertemporally dependent class, and Assumption U3 amounts to the requirement of concavity of $u$ in $(c, z)$, and strict concavity in $c$.

Finally, as will be shown in footnote 5 below, Assumption U4 (which, given Assumption U1, is always satisfied whenever $u$ happens to be an increasing function of $z$ ) guarantees that a uniformly maintained increase in the level of consumption along a balanced growth path will increase utility.

There is only one good, which can be either consumed or invested, and whose output at each point in time is the result of the linear production function

$$
y(t)=A k(t),
$$

where $y$ and $k$ are per-capita output and capital, respectively, and $A$ is a positive constant. ${ }^{3}$ We assume that individuals directly operate the economy's technology. Omitting from now on time indices whenever this choice does not risk confusion, it follows that the representative agent faces the budget constraint:

$$
\dot{k}=A k-c,
$$

where, for simplicity, depreciation of physical capital has been assumed away — or incorporated in $A$. Constraint (5) captures the fact that, in the closed economy with no outside assets and identical individuals we are about to study, capital accumulation is the only possible use of savings.

Finally, in order to be able to retrieve the standard " $A k$ "-results as a special case of our model, we also assume:

\section{T1. $A>\delta$.}

\footnotetext{
${ }^{3}$ Obviously this could be interpreted as a reduced form production function, which captures the essence of "convex" models of endogenous growth. For instance the same results obtained below would be valid by allowing capital to differ between physical an human capital, and with the ratio of the initial endowments of the two types of capital equal to the steady state one. If this ratio happened not to be the steady state one, it would induce further transitional dynamics.
} 


\section{Equilibrium}

Given the above definitions and assumptions, we have the following definitions of optimal allocation of resources and balanced-growth equilibrium, respectively.

Definition 1. An optimal allocation of resources is a set of paths $\{c(t), z(t)$, $k(t)\}$ that solve the maximization problem:

$$
\begin{aligned}
& \max U(c, z) \\
& \text { s.t. } \dot{k}=A k-c, \quad k(0)=k_{0}>0 \quad \text { given, } \\
& \dot{z}=\rho(c-z), \quad z(0)=z_{0}>0 \quad \text { given } .
\end{aligned}
$$

Definition 2. A balanced, or steady-state, equilibrium is a solution $\{c(t)$, $z(t), k(t)\}$ to the optimization problem (P1) such that $c(t), z(t)$ and $k(t)$ grow at a constant rate $g>0$.

Equipped with these definitions, in Appendix 1 we prove the following

Proposition 3. Given Assumptions U1 through U4, and T1, a sufficient condition for constant, positive steady-state growth is homogeneity of degree $\nu<1$ of the instantaneous felicity function $u$. The degree of homogeneity $\nu$ and the steady-state growth rate $g$ of the economy will be related according to:

$$
g=\frac{A-\delta}{1-\nu}
$$

That $c, k$ and $z$ will grow at a common rate $g$ in balanced equilibrium, as stated in Definition 2, can be readily verified by dividing the laws of motion of physical capital and habits by $k$ and $z$ respectively, and noting that the resulting growth rates of these variables will be constant if and only if the ratios $c / k$ and $c / z$ are also constant. As seems to be the rule in growth models where the utility function depends on a stock variable, Proposition 1 states that homogeneity of the instantaneous felicity function of the degree $\nu$ implicitly defined by equation (6) is a sufficient condition for 
a balanced growth path to qualify as an equilibrium. ${ }^{4,5}$ In the proof of this Proposition given in Appendix 1, we also show that, when the economy evolves along this balanced path, one must have

$$
g<A
$$

for the transversality conditions associated with problem (P1) to be satisfied. In other words, the steady state growth rate has to be less than the maximum "sustainable" rate that would be associated with zero consumption (see (5)).

Finally, it should be noted that, although Proposition 1 gives conditions under which a balanced path qualifies as an equilibrium, it does not imply that the economy will ever converge to it. In the next Section, we will place on $u$ additional restrictions to make sure that the economy asymptotically approaches a steady state with constant, positive growth.

\section{The Transformed Problem}

Given the above results, from now on we shall assume an instantaneous utility function homogenous of degree $\nu<1$. This assumption also allows us to reformulate problem (P1) in a way that greatly simplifies the analysis, and to give a graphical representation of the equilibrium evolution of the economy. To this end, following Caballé and Santos (1993), we introduce the normalized variables

$$
\begin{aligned}
\tilde{c}(t) & =c(t) e^{-g t}, \\
\tilde{z}(t) & =z(t) e^{-g t}, \\
\tilde{k}(t) & =k(t) e^{-g t} .
\end{aligned}
$$

\footnotetext{
${ }^{4}$ For an example of a model that resorts to the assumption of homogeneity of the instantaneous felicity function in a growth setting, see the one with endogenous leisure choice proposed by Rebelo (1991) along the lines of Heckman (1976). In that model, the momentary utility function depends on human capital; in our case, the stock variable on which instantaneous utility depends is consumption experience. The two commonly used intertemporally dependent specifications of preferences, the one by Abel (1990) and Carroll et al. (1997) in which the stock of habits enters the utility function multiplicatively, and the subtractive formulation introduced by Constantinides (1990), are both homogeneous.

${ }^{5}$ In the standard case of time separable preferences the restriction $v<1$ collapses to the assumption of concavity of the consumption function (Carroll and Kimball, 1996). Furthermore, if the model is one for which in equilibrium $c=z$, this restriction boils down to concavity of the utility function in $c=z$.
} 
These new variables will remain constant along a balanced path, and $\left\{\tilde{c}^{*}, \tilde{z}^{*}, \tilde{k}^{*}\right\}$ will denote their steady-state, balanced growth values. They will also be referred to as "de-trended" variables, since the normalization factor $e^{-g t}$ removes from the non-normalized ones the exponential growth trend that these latter will exhibit in a balanced equilibrium.

Next, we exploit the degree- $\nu$ homogeneity of $u$ to transform (1) into a function of $(\tilde{c}, \tilde{z})$ :

$$
U(\tilde{c}, \tilde{z})=\int_{0}^{\infty} e^{-\tilde{\delta} t} u(\tilde{c}, \tilde{z}) d t
$$

where

$$
\tilde{\delta}=\delta-g \nu
$$

Writing the dynamic constraints in terms of de-trended consumption, habits, and physical capital, we are in a position to reformulate (P1) as follows:

$$
\begin{array}{llll}
\max & U(\tilde{c}, \tilde{z}) & & \\
\text { s.t. } & \dot{\tilde{k}}=(A-g) \tilde{k}-\tilde{c}, & \tilde{k}_{0}=k_{0}>0 & \text { given, } \\
& \dot{\tilde{z}}=\rho \tilde{c}-(\rho+g) \tilde{z}, & \tilde{z}_{0}=z_{0}>0 & \text { given },
\end{array}
$$

and to write the corresponding current-value Hamiltonian function:

$$
\tilde{H}=u(\tilde{c}, \tilde{z})+\tilde{\lambda}[(A-g) \tilde{k}-\tilde{c}]+\tilde{\mu}[\rho \tilde{c}-(\rho+g) \tilde{z}] .
$$

The necessary conditions:

$$
\begin{aligned}
& u_{\tilde{c}}+\rho \tilde{\mu}=\tilde{\lambda}, \\
& \dot{\tilde{\lambda}}=(\tilde{\delta}-A+g) \tilde{\lambda}, \\
& \dot{\tilde{\mu}}=(\tilde{\delta}+\rho+g) \tilde{\mu}-u_{\tilde{z}},
\end{aligned}
$$

are, with the laws of motion of $\tilde{k}$ and $\tilde{z}$, also sufficient for a maximum if the following transversality conditions are met:

$$
\begin{aligned}
& \lim _{t \rightarrow \infty} e^{-\tilde{\delta} t} \tilde{\lambda}(t) \tilde{k}(t)=0, \\
& \lim _{t \rightarrow \infty} e^{-\tilde{\delta} t} \tilde{\mu}(t) \tilde{z}(t)=0 .
\end{aligned}
$$


While the co-state variable $\tilde{\lambda}$ is the shadow value of normalized capital, $\tilde{\mu}$, that — from $(11)$ - can be written as

$$
\tilde{\mu}(t)=\int_{t}^{\infty} e^{-(\tilde{\delta}+\rho+g)(s-t)} u_{\tilde{z}}(\tilde{c}(s), \tilde{z}(s)) d s,
$$

is the shadow value of an additional unit of $\tilde{z}$. Condition (9) implies that, along an optimal path, at each time $t$ the current marginal utility of consumption, plus the contribution of greater time- $t$ consumption to the utility stream derived from future consumption experience - a contribution that is positive if $u_{\tilde{z}}>0$, and negative in the opposite case - must be equal to the time- $t$ shadow value of capital. We define the sum $\left(u_{\tilde{c}}+\rho \tilde{\mu}\right)$ "the time- $t$ full marginal benefit of $\tilde{c}$ " to distinguish the present setting from the standard time-independent case, where the contribution of greater time- $t$ consumption to the objective functional is given by the term $u_{\tilde{c}}$ only.

In the steady state, condition (10) requires

$$
\tilde{\delta}=A-g,
$$

a positive quantity by (7). Using (8), it follows that:

$$
g=\frac{A-\delta}{1-\nu}
$$

an expression that gives the same steady state growth rate of the economy derived in Proposition 1. From these results, it can be immediately verified that $\tilde{\lambda}$ will be constant at all times, at a level that we shall denote by $\tilde{\lambda}^{*}$ and whose expression will be derived below.

The differentiation of (9) with respect to time, using (10) and (11) and taking into account the laws of motion of $\tilde{z}$ and $\tilde{k}$, results in the following autonomous system of differential equations in $(\tilde{c}, \tilde{z}, \tilde{k})$ :

$$
\begin{aligned}
& \dot{\tilde{c}}=\left(\frac{1}{u_{\tilde{c} \tilde{c}}}\right) \cdot\left\{(A+\rho)\left(u_{\tilde{c}}-\tilde{\lambda}^{*}\right)+\rho u_{\tilde{z}}-u_{\tilde{c} \tilde{z}}[\rho \tilde{c}-(\rho+g) \tilde{z}]\right\}, \\
& \dot{\tilde{z}}=\rho \tilde{c}-(\rho+g) \tilde{z} \\
& \dot{\tilde{k}}=(A-g) \tilde{k}-\tilde{c} .
\end{aligned}
$$

From the last two equations, the steady state levels of the detrended habits and capital are:

$$
\begin{aligned}
& \tilde{z}^{*}=\left(\frac{\rho}{\rho+g}\right) \cdot \tilde{c}^{*}, \\
& \tilde{k}^{*}=\left(\frac{1}{A-g}\right) \cdot \tilde{c}^{*} .
\end{aligned}
$$


To derive $\tilde{c}^{*}$, notice that (14a) implies that, in the steady state,

$$
u_{\tilde{c}}\left(\tilde{c}^{*}, \tilde{z}^{*}\right)+\frac{\rho}{A+\rho} u_{\tilde{z}}\left(\tilde{c}^{*}, \tilde{z}^{*}\right)=\tilde{\lambda}^{*}
$$

a positive quantity ${ }^{6}$. Being $\left(\tilde{z}^{*} / \tilde{c}^{*}\right)=\left(\frac{\rho}{\rho+g}\right)$, homogeneity of degree $(\nu-1)$ of $u_{\tilde{c}}$ and $u_{\tilde{z}}$ implies that the left hand side of the above equation is equal to $\left(\tilde{c}^{*}\right)^{\nu-1} \cdot\left[u_{\tilde{c}}\left(1, \frac{\rho}{\rho+g}\right)+\left(\frac{\rho}{A+\rho}\right) \cdot u_{\tilde{z}}\left(1, \frac{\rho}{\rho+g}\right)\right]$, so that:

$$
\tilde{c}^{*}=\left[\frac{\tilde{\lambda}^{*}}{u_{\tilde{c}}\left(1, \frac{\rho}{\rho+g}\right)+\left(\frac{\rho}{A+\rho}\right) \cdot u_{\tilde{z}}\left(1, \frac{\rho}{\rho+g}\right)}\right]^{\frac{1}{\nu-1}} .
$$

We show in Appendix 2 that $\tilde{c}^{*}$, and therefore $\tilde{\lambda}^{*}, \tilde{z}^{*}$ and $\tilde{k}^{*}$, are uniquely pinned down by the need to satisfy the transversality conditions (12), given the initial conditions on the state variables. This result, and the assumptions placed on $u$, imply that the steady-state equilibrium just derived is unique ${ }^{7}$.

To investigate the dynamic evolution of the economy and the stability properties of the steady state just characterized, in Appendix 2 we linearize system (14) around the steady state (15), and show that this latter is a saddlepoint provided that

$$
j \equiv-\frac{(A+g+2 \rho) u_{\tilde{c} \tilde{z}}^{*}+\rho u_{\tilde{z} \tilde{z}}^{*}}{u_{\tilde{c} \tilde{c}}^{*}}<\frac{(A+\rho)(\rho+g)}{\rho},
$$

where starred derivatives are evaluated at the steady state.

The crucial role played by the sign and size of $j$ in determining the dynamic evolution of consumption and habits in a model with intertemporally dependent preferences was first pointed out by Ryder and Heal (1973). In

\footnotetext{
${ }^{6}$ This is also true when the marginal utility of habits happens to be negative. In this case, since $g<A$, assumption U4 implies that $u_{\tilde{c}}^{*}+\left(\frac{\rho}{A+\rho}\right) u_{\tilde{z}}^{*}>u_{\tilde{c}}^{*}+\left(\frac{\rho}{\rho+g}\right) u_{\tilde{z}}^{*}>0$.

${ }^{7}$ Evaluated at this steady state, instantaneous utility is $u\left(\tilde{c}^{*}, \tilde{z}^{*}\right)=u\left(\tilde{c}^{*},\left(\frac{\rho}{\rho+g}\right) \tilde{c}^{*}\right)$. The derivative of this expression with respect to $\tilde{c}^{*}$ is $u_{\tilde{c}}^{*}+\left(\frac{\rho}{\rho+g}\right) u_{\tilde{z}}^{*}$, which is positive by Assumption U4. To see what this latter implies, let us assume that the economy is on a balanced growth path, with consumption and habits growing over time at a constant, positive rate $g$, and consider the two sequences $\{c(t), z(t)\}_{t=s}^{\infty}$ and $\left\{c^{\prime}(t), z^{\prime}(t)\right\}_{t=s}^{\infty}$, with $c^{\prime}(s)>c(s)$ and $\dot{c}(t) / c(t)=\dot{c}^{\prime}(t) / c^{\prime}(t)=g, t=s, \ldots, \infty$. Assumption U4 amounts to the (in our opinion, sensible) requirement that the second sequence will yield greater utility to the individual. Notice that this assumption is the generalization to a growth setting of the non-satiation condition in Ryder and Heal [1973, p.3].
} 
their terminology, one has "adjacent complementarity" — that is, complementarity between consumption at adjacent dates, a property of preferences that Becker and Murphy (1988) identify with addiction — if $j>0$, and "distant complementarity" if $j<0$. Notice that assumption U3 implies that $j$ is always negative if $u_{\tilde{c} \tilde{z}}<0$. To have $j>0$, one needs $u_{\tilde{c} \tilde{z}}>0$ and large enough. When this is the case, condition (16) places an upper bound on the degree of adjacent complementarity consistent with saddlepath stability of system (15). We show in Appendix 2 that values of $j$ that violate condition (16) lead to instability, or to a violation of the hypothesis of concavity of $u$; both instances are ruled out by assumption in the present analysis, so that (16) always holds.

For this case, in the same Appendix we show that equilibrium-normalized consumption, habits and physical capital evolve according to:

$$
\begin{aligned}
& \tilde{c}(t)-\tilde{c}^{*}=\left[\tilde{c}_{0}-\tilde{c}^{*}\right] \cdot e^{-\psi t}, \\
& \tilde{z}(t)-\tilde{z}^{*}=\omega_{1} \cdot\left[\tilde{c}(t)-\tilde{c}^{*}\right], \\
& \tilde{k}(t)-\tilde{k}^{*}=\omega_{2} \cdot\left[\tilde{c}(t)-\tilde{c}^{*}\right],
\end{aligned}
$$

where $-\psi$ is the negative, real characteristic root associated with the linearized version of system (14), and

$$
\omega_{1} \equiv \frac{\rho}{\rho+g-\psi}, \quad \omega_{2} \equiv \frac{1}{A-g+\psi} .
$$

While $\omega_{2}>0$ always, in Appendix 2 we prove that $\omega_{1}$ has the same sign as $j$. We also show that steady state de-trended consumption is given by:

$$
\tilde{c}^{*}=\frac{(A-g)(\rho+g)}{\psi(A+\rho)}\left[-\frac{1}{\omega_{1}} z_{0}+\frac{1}{\omega_{2}} k_{0}\right],
$$

while $\tilde{z}^{*}$ and $\tilde{k}^{*}$, which are increasing in $\tilde{c}^{*}$, can be computed using (18) in equations (15a) and (15b). ${ }^{8}$ Finally, the difference between optimal

\footnotetext{
${ }^{8}$ When complementarity is adjacent $-\omega_{1}, j>0-$ the linearization imposes an upper bound on the value that the ratio of initial conditions may take on. Namely, and as is clear from (18), for an optimal program to exist, $\left(z_{0} / k_{0}\right)$ has to be less than $\left(z_{0} / k_{0}\right)^{\max }=\rho[(A-g+\psi) /(\rho+g-\psi)]$. Values of $\left(z_{0} / k_{0}\right)$ above this quantity imply so much consumption at time $t=0$ that $\tilde{c}, \tilde{k}, \tilde{z}$ become zero in finite time. In terms of the diagrams we introduce in Section 5 , for a given $k_{0}$ one finds the maximum stock of initial habits consistent with the existence of an equilibrium as the value of $z_{0}$ that, in Figure 2, generates a saddlepath $S S^{\prime}$ in the upper quadrant crossing the $\dot{\tilde{z}}=0$ locus at $(\tilde{z}, \tilde{c})=(0,0)$.
} 
time-0 and steady-state normalized consumption that appears in (17a) can be written as:

$$
\tilde{c}_{0}-\tilde{c}^{*}=\frac{(\rho+g)}{\psi(A+\rho) \omega_{1} \omega_{2}} \cdot\left[z_{0}-\frac{\rho(A-g)}{(\rho+g)} k_{0}\right] .
$$

Equations (17)-(19) imply the following facts about the equilibrium dynamics associated with the solution of the transformed problem (P1')

Proposition 4. In equilibrium:

(i) normalized consumption, habits and physical capital converge monotonically over time to the steady state $\left\{\tilde{c}^{*}, \tilde{z}^{*}, \tilde{k}^{*}\right\}$;

(ii) the steady-state levels of the same variables are decreasing in $z_{0}$ under adjacent complementarity $(j>0)$, and increasing in $z_{0}$ under distant complementarity $(j<0)$; independently of the sign of $j, \tilde{c}^{*}, \tilde{z}^{*}$ and $\tilde{k}^{*}$ increase with $k_{0}$;

(iii) when $j>0$, normalized consumption increases (decreases) over time toward its steady-state level if $\frac{z_{0}}{k_{0}}<(>) \frac{\rho(A-g)}{\rho+g}$. The opposite conclusion holds when $j<0$;

(iv) in the transition to the steady state, $\tilde{c}$ and $\tilde{k}$ will always covary positively; normalized consumption and habits $\tilde{z}$ will covary positively if $j>0$, and negatively if $j<0$.

\section{$5 \quad$ Picturing Transitional Dynamics}

In this Section, we modify and extend a technique used by Obstfeld (1992) and draw a simple diagram to illustrate the transitional dynamics implied by our model and to provide the economic intuition for the results derived so far, and summarized in Proposition 2. Although we choose to focus on the case of adjacent complementarity, which we regard as most relevant, the same arguments can be used to give an account of the dynamic evolution of the variables in the model when complementarity is distant.

Assuming $j>0$, in Figure 1 we draw four loci:

- the $(\dot{\tilde{z}}=0)$-locus, which — see equation $(14 \mathrm{~b})$ - is a straight line emanating from the origin with slope $(\rho+g) / \rho>1$ in the $(\tilde{z}, \tilde{c})$-plane; $\tilde{z}$ will be increasing over time above this locus, and decreasing below it; 
- the stable saddle path in the same plane, obtained by combining equations (17a) and (17b); in the upper quadrant, it is the arrowed path labeled $S S^{\prime}$, with slope $(\rho+g-\psi) / \rho$, positive and smaller than the slope of the $\dot{\tilde{z}}=0$ locus;

- the saddlepath in the $(\tilde{k}, \tilde{z})$-plane, the line $T T^{\prime}$ with slope $\frac{\rho(A-g+\psi)}{\rho+g-\psi}$, obtained by combining equations (17b) and (17c);

- the relationship between steady state levels of normalized habits and physical capital that is implied by (14a) and (14b), the straight line emanating from the origin in the lower quadrant, with slope $\frac{\rho(A-g)}{\rho+g}$ $\left(<\frac{\rho(A-g+\psi)}{\rho+g-\psi}\right)$.

It should be noted that those shown in the figure are not standard phase diagrams. This is because the steady state levels of $\tilde{c}, \tilde{z}$ and $\tilde{k}-$ and, with them, the location of the two saddlepaths $S S^{\prime}$ and $T T^{\prime}$ - depend on the set of initial conditions $\left(z_{0}, k_{0}\right)$, as is clear from (17)-(19).

To understand how this graphical device helps determine the equilibrium dynamics for arbitrary initial conditions on the stock variables, let us assume that the economy starts off with the pair $\left(z_{0}, k_{0}\right)$ given by point $T$ in the lower quadrant of the figure. Notice that the assumed configuration of initial conditions is such that $\left(z_{0} / k_{0}\right)<\frac{\rho(A-g)}{\rho+g}$. The steady state pair $\left(\tilde{k}^{*}, \tilde{z}^{*}\right)$ - point $T^{\prime}$ - is found as the intersection between the line emanating from $T$ with slope $\frac{\rho(A-g+\psi)}{\rho+g-\psi}$ and the steady state locus $\tilde{z}^{*}=\frac{\rho(A-g)}{\rho+g} \tilde{k}^{*}$. Given the $\tilde{z}^{*}$ so determined, one uses the $(\dot{\tilde{z}}=0)$-locus in the upper quadrant to find the pair $\left(\tilde{z}^{*}, \tilde{c}^{*}\right)$ - point $S^{\prime}$. The saddlepath in the $(\tilde{z}, \tilde{c})$-plane is then the line going through $S^{\prime}$ with slope $\left(1 / \omega_{1}\right) \equiv \frac{\rho+g-\psi}{\rho} \quad$ (a positive quantity, under adjacent complementarity); finally, one determines the optimal time- 0 choice of consumption, $\tilde{c}_{0}$, as the value of $\tilde{c}$ that, along this line, is associated with the assumed $z_{0}$.

From the figure, it is clear that, as stated in Proposition 1, a stable dynamics calls for levels of normalized consumption, habits, and physical capital to rise over time. In fact, given $\left(z_{0} / k_{0}\right)<\frac{\rho(A-g)}{\rho+g}$, optimal time-0 consumption is lower than the level $(A-g) k_{0}$ which - through (14c) would yield $\dot{\tilde{k}}(0)=0$, and point $S$ is located above the $(\dot{\tilde{z}}=0)$-locus. It follows that both normalized physical capital and habits will be increasing at time zero. The next instant - which, for simplicity, we call $t=1-$ the economy will therefore start off with larger beginning-of-period $\tilde{k}$ and $\tilde{z}$. All other things being equal, a larger capital stock will exert a positive 
wealth effect on time- 1 consumption, $\tilde{c}_{1}$. In addition, under adjacent complementarity the individual has a further incentive to raise his consumption level at time $t=1$ because of the increase in the stock of habits. For both reasons, $\tilde{c}_{1}>\tilde{c}_{0}$.

To understand why an increase in $\tilde{z}$ leads to an increase in the optimal choice of $\tilde{c}$, notice that (13) implies that what we have termed the "full marginal benefit" of current consumption can be written as:

$$
u_{\tilde{c}}(\tilde{c}(t), \tilde{z}(t))+\rho \int_{t}^{\infty} e^{-(A+\rho)(s-t)} u_{\tilde{z}}(\tilde{c}(s), \tilde{z}(s)) d s .
$$

Since, for $s>t$, one has

$$
\tilde{z}(s)=\tilde{z}(t) e^{-(\rho+g)(s-t)}+\rho \int_{t}^{s} e^{-(\rho+g)(s-\tau)} c(\tau) d \tau,
$$

the derivative with respect to $\tilde{z}(t)$ of $(20)$ is ${ }^{9}$ :

$$
u_{\tilde{c} \tilde{z}}(\tilde{c}(t), \tilde{z}(t))+\rho \int_{t}^{\infty} e^{-(A+g+2 \rho)(s-t)} u_{\tilde{z} \tilde{z}}(\tilde{c}(s), \tilde{z}(s)) d s .
$$

Evaluated at the steady state, (21) reduces to

$$
u_{\tilde{c} \tilde{z}}^{*}+\frac{\rho}{A+g+2 \rho} u_{\tilde{z} \tilde{z}}^{*} \equiv\left(-u_{\tilde{c} \tilde{c}}^{*}\right) \cdot(A+g+2 \rho) \cdot j,
$$

an expression which has the same sign as $j$. It follows that, in the local analysis of the equilibrium dynamics under adjacent complementarity we are carrying out, the full marginal benefit of $\tilde{c}$ will move in the same direction as $\tilde{z}$, and the individual has an incentive to increase $\tilde{c}$ when $\tilde{z}$ rises.

Having shown that, for the assumed configuration of initial conditions, $\tilde{c}_{1}>\tilde{c}_{0}$, it is straightforward to verify that, at time $t=1$, this higher level of consumption is still consistent with the accumulation of capital and habits, although at a slower rate than in the previous period. The same process is repeated the next instant and the economy converges over time to the steady state $\left(S^{\prime}, T^{\prime}\right)$ along the arrowed paths in the two quadrants.

Suppose now that the economy starts off with an unchanged level of physical capital, but with a $z_{0}^{\prime}>z_{0}$. If , as assumed in the figure, this increase in initial consumption experience is such that $\left(z_{0}^{\prime} / k_{0}\right)$ is still less than the critical level $\frac{\rho(A-g)}{\rho+g}$, we end up with the new saddlepaths given

\footnotetext{
${ }^{9}$ This step involves the computation of the "Volterra derivative" of the functional in (20). For a definition of Volterra derivatives, see Ryder and Heal (1973), pp. 3-4.
} 
by the dashed lines labeled $S^{\prime \prime} S^{\prime \prime \prime}$ and $T^{\prime \prime} T^{\prime \prime \prime}$, and with lower steady state levels of $\tilde{c}, \tilde{z}$ and $\tilde{k}$.

That the steady state levels of the variables are decreasing in $z_{0}$ when $j>0$ simply reflects the higher marginal benefit of consumption associated with higher initial habits. The individual will consume more at time 0 , and will accumulate less capital ${ }^{10}$. This smaller accumulation will — via a wealth effect - cause a smaller increase in consumption, and therefore habits, during the transition to the steady state, as well as lower levels of the variables in the new balanced growth equilibrium $\left(S^{\prime \prime \prime}, T^{\prime \prime \prime}\right)$.

On the other hand, when $z_{0}$ happens to be so large that $\left(z_{0} / k_{0}\right)>$ $\frac{\rho(A-g)}{\rho+g}$, the whole dynamics is reversed. As shown in Figure 1, under adjacent complementarity the individual will choose to consume so much at time zero that $\tilde{k}$ will be decumulated $\left(\tilde{c}_{0}>(A-g) k_{0}\right)$. De-trended habits will decrease as well, since the economy starts off at point $S$, which is now below the $\dot{\tilde{z}}=0$ locus: although the individual consumes a lot, the optimal initial choice of consumption - one that is consistent with the transversality condition on $\tilde{k}$ - does not add to consumption experience enough to compensate for the depreciation term $(\rho+g) z_{0}$, which is large because $z_{0}$ is large. In this case, normalized consumption, habits and physical capital will decrease over time toward their steady-state levels.

The same diagram can be used to determine the effects of changes in $k_{0}$ for a given $z_{0}$. For instance, and going back to Figure 1, an increase in $k_{0}$ would cause a parallel, downward shift of the $T T^{\prime}$ locus and an upward shift of the $S S^{\prime}$ locus, thus leading to an increase both in the initial optimal choice of $\tilde{c}$, and in the steady state levels of the three variables on the axes. If the initial configuration of initial conditions is the one shown in Figure 2 , an increase in $k_{0}$ such that the ratio $\left(z_{0} / k_{0}\right)$ remains above the critical value $\frac{\rho(A-g)}{\rho+g}$ leads to qualitatively similar displacements of the $T T^{\prime}$ and the $S S^{\prime}$ loci, and, once again, to higher steady state values of $\tilde{c}^{*}, \tilde{z}^{*}$, and $\tilde{k}^{*}$.

\footnotetext{
${ }^{10}$ That $\tilde{c}_{0}$ is increasing in $\tilde{z}_{0}$ simply reflects the fact that consumption is increasing in habits under adjacent complementarity. It follows that, as shown in the Figure, point $S^{\prime}$ is located to the north-east of point $S$. This can be proved as follows. First, evaluate at time $t=0$ the expressions for the two loci $T T$ ' and $S S^{\prime}$, obtaining $\quad \tilde{z}_{0}-\tilde{z}^{*}=\frac{\rho(A-g+\psi)}{(\rho+g-\psi)}\left(\tilde{k}_{0}-\tilde{k}^{*}\right)$ and $\tilde{c}_{0}-\tilde{c}^{*}=\frac{(\rho+g-\psi)}{\rho}\left(\tilde{z}_{0}-\tilde{z}^{*}\right)$. Next, differentiate totally the first expression, setting $d \tilde{k}_{0}=0$ and $d \tilde{k}^{*}=\frac{(\rho+g)}{\rho(A-g)} d \tilde{z}^{*}$, to get $d \tilde{z}^{*}=-\frac{(A-g)(\rho+g-\psi)}{\psi(A+\rho)} d \tilde{z}_{0}$. Finally, differentiation of the second expression, using $d \tilde{c}^{*}=\frac{(\rho+g)}{\rho} d \tilde{z}^{*}=-\frac{(\rho+g)(A-g)(\rho+g-\psi)}{\rho \psi(A+\rho)} d \tilde{z}_{0}$, yields $d \tilde{c}_{0} / d \tilde{z}_{0}=\frac{(\rho+g)(\rho+g-\psi)}{\rho(A+\rho)}>0$. The same result can be derived, in a more straightforward fashion, using the explicit expresion for $\tilde{c}_{0}$ given by (A.2.12) in Appendix 2 .
} 
More generally, to assess the impact of simultaneous changes of $k_{0}$ and $z_{0}$, or the qualitative properties of the transitional dynamics of the variables starting from an arbitrary pair $\left(z_{0}, k_{0}\right)$, all that matters is how the ratio $\left(z_{0} / k_{0}\right)$ compares to $\frac{\rho(A-g)}{\rho+g}-$ or, in graphical terms, whether the point that denotes the initial conditions on the two stock variables in the lower quadrant of the Figure is located above or below the locus $\tilde{z}^{*}=\frac{\rho(A-g)}{\rho+g} \tilde{k}^{*}$. If, by accident, $\left(z_{0} / k_{0}\right)=\frac{\rho(A-g)}{\rho+g}$, the economy jumps immediately on the steady state. In general, however, consumption, habits and physical capital converge to a balanced growth path increasing or decreasing over time, depending on whether $\left(z_{0} / k_{0}\right) \lessgtr \frac{\rho(A-g)}{\rho+g}$.

Finally, the results one gets under the standard assumption of timeseparable preferences can be retrieved as a special case of our model.

To see this, first notice that, if $u_{\tilde{z}}=u_{\tilde{c} \tilde{z}}=0$, so that Assumption U2 is violated, one has $\tilde{\mu}=0, u_{\tilde{c}}=\tilde{\lambda}^{*}, \forall t$. It follows that the right-hand side of equation (14a) is zero, and the saddlepaths $S S^{\prime}$ and $T T^{\prime}$ become flat at the levels of consumption $\tilde{c}=(A-g) k_{0}$ and capital $\tilde{k}=k_{0}$, respectively. Regardless of initial conditions, this implies that de-trended consumption and physical capital will be constant over time, and that - as in the standard " $A k$ " model $-c$ and $k$ will always grow at the steady-state rate $g=\frac{A-\delta}{1-\nu}$. Given the usual time-separable, isoelastic instantaneous felicity function $\frac{c^{1-\sigma}}{1-\sigma}$, which is homogeneous of degree $\nu=1-\sigma$, this is just the familiar growth rate $\frac{A-\delta}{\sigma} \cdot{ }^{11}$

Up to this point, we have provided a full characterization of the equilibrium dynamics of what we have termed "normalized", or "de-trended", variables. In order to go from the latter to the behavior over time of "actual" consumption, habits, capital, and output, one has simply to remember that the generic variable $x$ is related to its normalized counterpart $\tilde{x}$ according to $x=e^{g t} \tilde{x}$, or, in terms of growth rates:

$$
\frac{\dot{x}}{x}=g+\frac{\dot{\tilde{x}}}{\tilde{x}}
$$

Since normalized variables converge monotonically over time to a steady state where they take on constant values, the growth rate of the actual ones will converge asymptotically to $g$. In the transition, their growth rate will be above or below this value, depending on whether their de-trended

\footnotetext{
${ }^{11}$ If, for given $k_{0}, z_{0} \neq \frac{\rho(A-g)}{\alpha+g} k_{0}$, there will be a transitional dynamics of the stock of habits; however, this will not affect consumption, capital, nor utility levels.
} 
counterparts converge to the steady state increasing or decreasing over time - an information one can readily retrieve from Proposition 2, or the first row of Table 1.

In turn, it is possible to infer the behavior of the growth rate of percapita output, $g_{y}$, by noticing that:

$$
g_{y} \equiv \frac{\dot{y}}{y}=\frac{\dot{k}}{k}=g+\frac{\dot{\tilde{k}}}{\tilde{k}} .
$$

Using these results, the last two rows of Table 1 summarize the transitional dynamics of $g_{y}$ under adjacent complementarity. The growth rate of per-capita output is decreasing in $\left(z_{0} / k_{0}\right)$, and can initially be negative for values of this ratio that are very high, while still being consistent with the upper bound mentioned in footnote $8 .{ }^{12}$ For values of $\left(z_{0} / k_{0}\right)$ below (above) the threshold $\frac{\rho(A-g)}{(\rho+g)}, g_{y}$ will be larger (smaller) than $g$, converging asymptotically to this constant, positive value.

Table 1: Transitional dynamics

\begin{tabular}{l|c|c}
\hline \hline & $\frac{z_{0}}{k_{0}}<\frac{\rho(A-g)}{(\rho+g)}$ & $\frac{z_{0}}{k_{0}}>\frac{\rho(A-g)}{(\rho+g)}$ \\
\hline$\dot{\tilde{c}}, \dot{\tilde{z}}, \dot{\tilde{k}}$ & $>0$ & $<0$ \\
$g_{y}$ & $>g$ & $<g$ \\
$\dot{g}_{y}$ & $<0$ & $>0$ \\
\hline
\end{tabular}

Finally, defining the saving rate as:

$$
s \equiv 1-\frac{c}{A k},
$$

and noticing that $(c / k)=(\tilde{c} / \tilde{k})=(A-g)-(\dot{\tilde{k}} / \tilde{k})$, from $(22)$ one has:

$$
s=\frac{g_{y}}{A} .
$$

It follows that $s$ will take on the constant value $s^{*}=(g / A)<1$ in balanced growth, and that its transitional dynamics will be qualitatively identical

\footnotetext{
${ }^{12}$ Since $g_{y}$ asymptotically approaches $g>0, g_{y}<0$ is possible only during the first stages of the transition. Furthermore, the possibility of a negative growth rate depends not just on the size of the ratio $\left(z_{0} / k_{0}\right)$, but also - through $j$ and $\psi$ - on the characteristics of the instantaneous utility function.
} 
to that of the rate of growth of per-capita output: the saving rate will be initially "high", and decreasing over time, when $\frac{z_{0}}{k_{0}}<\frac{\rho(A-g)}{(\rho+g)}$, and relatively "low", but increasing toward its steady state level, for the opposite configuration of initial conditions.

\section{An example, with implications for distribution dynamics}

In this Section we consider a particular specification of preferences belonging to the intertemporally dependent class, proposed by Constantinides (1990) in his attempt to solve the equity premium puzzle and used, among others, by Detemple and Zapatero (1991). We, further, assume heterogeneity in the distribution of initial habits and capital stock and, having shown that the average of all agents behaves exactly as if there was a single agent, with average initial capital and habits, we analyze the predictions of the model with respect to the evolution of the cross-section of per capita capital holdings. We also compare the implications of our model with those associated with a different departure from the assumption of CIES preferences.

Consider the functional form:

$$
\begin{aligned}
u\left(c_{i}, z_{i}\right) & =\frac{\left(c_{i}-b z_{i}\right)^{1-\sigma}}{1-\sigma}, & & \text { for } c_{i} \geq b z_{i}, \\
& =-\infty & & \text { for } c_{i}<b z_{i},
\end{aligned}
$$

where the subscript $i$ denotes the generical individual; $\sigma>0$ and $\neq 1$, $1 \geq b \geq 0$. We also assume that at time zero each agent is endowed with enough capital to produce $b z_{0, i}$, that is $A k_{0, i}>b z_{0, i} \cdot{ }^{13}$ Since $\lim _{c_{i}-b z_{i} \rightarrow 0} u_{c}=\infty$ and $z_{0, i}>0, \quad c_{t, i}>b z_{t, i}>0 \forall t \forall i$. In addition, $u_{z}, u_{c c}, u_{z z}<0$, $u_{c c} u_{z z}-u_{c z}^{2}=0$, assumption U4 is met and $j=b[A+g+\rho(2-b)]>0$, so we are always in the adjacent complementarity case.

Without loss of generality, and only to simplify the algebra, let us assume from here on $b \equiv 1$, which implies that only the excess of consumption over the standard of living is valued. In Appendix 3 we show that the capital

\footnotetext{
${ }^{13}$ Equivalently, the upper bound on the ratio of initial conditions mentioned in footnote 8 above now becomes $\left(z_{0, i} / k_{0, i}\right)<A / b$. Interesting complications would arise when, at least for some agents, this inequality is not satisfied, that is when some individuals are wealth constrained. These are not discussed in this paper, since they would lead us too far afield.
} 
stock of the $i$-th individual evolves according to:

$$
k_{t, i}=\left[\frac{\rho+g}{g(A+\rho)}\right]\left(A k_{0, i}-z_{0, i}\right) e^{g t}+\left[\frac{\rho+g}{g(A+\rho)}\right]\left[z_{0, i}-\left(\frac{\rho(A-g)}{\rho+g}\right) k_{0, i}\right],
$$

where $g=\frac{A-\delta}{\sigma}$ is the common steady state growth rate of each variable in the model. Since this equation is linear in $\left(k_{0, i}, z_{0, i}\right)$, it is immediate to note that the average of all agents (the representative consumer) behaves exactly as if there was a single agent, with average initial capital and habits $\left(k_{0}^{a}, z_{0}^{a}\right)$.

The growth rate of each agent's output and capital is:

$$
g_{y, i}=\frac{\dot{k_{t, i}}}{k_{t, i}}=\left(k_{t, i}\right)^{-1}\left(\frac{\rho+g}{A+\rho}\right)\left(A k_{0, i}-z_{0, i}\right) e^{g t}>0 .
$$

Notice that:

$$
\dot{g}_{y, i}=\left(k_{t, i}\right)^{-2}\left(\frac{\rho+g}{A+\rho}\right)^{2}\left(A k_{0, i}-z_{0, i}\right)\left[z_{0, i}-\frac{\rho(A-g)}{\rho+g} k_{0, i}\right] e^{g t},
$$

which is negative for $\left(z_{0, i} / k_{0, i}\right) \in\left(0, \frac{\rho(A-g)}{\rho+g}\right)$, and positive for $\left(z_{0, i} / k_{0, i}\right) \in$ $\left(\frac{\rho(A-g)}{\rho+g}, A\right)$.

The attractive property of the functional form under consideration is that these equilibrium paths represent the global dynamics of the variables, that one gets solving the system (14a)-(14c) directly (see Appendix 3). ${ }^{14}$

The model with intertemporally dependent preferences just outlined therefore allows us to study the dynamics of the distribution of per capita capital, which is totally absent in the the standard time separable specification (Rebelo, 1991). Of course, the simplicity of the model, especially for what concerns the production side of the economy, makes the following exercise purely illustrative of the potential implications of a richer specification of preferences and does not intend to provide a conving explanation of the actual determinants of the evolution of inequality. ${ }^{15}$

As in Caselli and Ventura (2000), this model, since it allows for a representative agent, cannot be used to study how the initial wealth distribution

\footnotetext{
${ }^{14}$ The same conclusion does not hold for the ratio specification of preferences of Abel (1990) and Carroll et al. (1997, 2000). In this case all the results we will derive below hold in the approximation around the steady state.

${ }^{15}$ Diaz et al. (forthcoming) show that habit formation is an important determinant of precautionary savings in economies with idiosyncratic uncertainty.
} 
affects economic growth. However, it can yield interesting insights on the evolution of the wealth distribution along the transition towards a steady state growth and it can be used to analyze the effect of growth on the steady state distribution. The effects of inequality on economic growth is surveyed in Benabou (1996). Aghion et al. (1999) also analyze the way in which growth might affect inequality and concludes that technical change, besides being one of the main sources of economic growth, is also one of the main determinants of the distribution of wages and therefore of inequality. Yet, the extent to which growth induces higher inequality is uncertain since it is mediated by the institutional characteristics of each country. Empirical evidence, arising from cross-country analyses, is not conclusive. In the only study we are aware of, Deininger and Squire (1996) find that episodes of growth were, almost with the same frequence, followed by increases or decreases in inequality. Moreover, and more importantly from the point of view of the present analysis, does not seem to emerge any systematic relationship between growth and subsequent inequality, even limiting to subgroups of countries such as advanced vs. low-middle income. As it will be clear below, this is consistent with our model, where the level of initial habits play a prominent role.

To study the evolution of inequality let us define the ratio of individial $i$ 's capital to the average one $k_{t}^{R} \equiv k_{t, i} / k_{t}^{a}$. This can be written as

$$
k_{t}^{R}=\frac{\left(A k_{0, i}-z_{0, i}\right)+\left[z_{0, i}-\left(\frac{\rho(A-g)}{\rho+g}\right) k_{0, i}\right] e^{-g t}}{\left(A k_{0}^{a}-z_{0}^{a}\right)+\left[z_{0}^{a}-\left(\frac{\rho(A-g)}{\rho+g}\right) k_{0}^{a}\right] e^{-g t}} .
$$

In steady state this ratio will be constant and will depend only on initial conditions, as well as on the productivity parameter $A$ :

$$
\left(k^{R}\right)^{*}=\frac{\left(A k_{0, i}-z_{0, i}\right)}{\left(A k_{0}^{a}-z_{0}^{a}\right)} .
$$

Yet, along the transition $k_{t}^{R}$ can either increase or decrease. We first study the evolution of inequality during the transitional dynamics, then we analyze how the steady state distribution modifies following a productivity shock.

First of all, notice that - assuming that the individual $i$ was initially richer than the mean $\left(k_{0, i}>k_{0}^{a}\right)$ - nothing precludes $\left(k^{R}\right)^{*}<1$, that is nothing precludes that he can become poorer than the mean. This will happen if $\left(z_{0, i}-z_{0}^{a}\right)>A\left(k_{0, i}-k_{0}^{a}\right)>0$. As described before, the fact that under 
adjacent complementarity the steady state level of capital is decreasing in $z_{0}$ reflects the higher marginal benefit of consumption associated with initial habits. With a very high habit stock the individual $i$ will chose a level of consumption so high, and a saving rate so low that he will end up poorer than the mean. Stretching this conclusion to the implications for the pattern of cross-country growth, if individuals are interpreted as countries, it means that this model can replicate growth miracles or disasters.

By differentiating $k_{t}^{R}$ with respect to time, after some algebra, it can be shown that along the transition the distribution of capital becomes more unequal (equal) if $k_{0}^{a} z_{0, i}-k_{0, i} z_{0}^{a}>0(<0)$. This means that an individual $i$ initially richer than the mean $\left(k_{0, i}>k_{0}^{a}\right)$ will increase (decrease) his distance from the mean if $k_{0, i} / z_{0, i}>(<) k_{0}^{a} / z_{0}^{a}$. To understand the intuition of this result, let us examine the initial saving rates ratio, which is given by

$$
s_{0}^{R} \equiv \frac{s_{0, i}}{s_{0}^{a}}=\frac{k_{0}^{a}\left(A k_{0, i}-z_{0, i}\right)}{k_{0, i}\left(A k_{0}^{a}-z_{0}^{a}\right)} .
$$

When $k_{0, i} / z_{0, i}>k_{0}^{a} / z_{0}^{a}, s_{0}^{R}>1$, which means that the individual $i$, already richer than the mean, saves also more than the mean, augmenting more than proportionally his capital stock and increasing the inequality. The reverse is true when $k_{0, i} / z_{0, i}<k_{0}^{a} / z_{0}^{a}$.

Now let us imagine a positive permanent shock to $A$. This will raise the steady state rate of growth, but how will it affect the steady state distribution of capital? Differentiating (25) with respect to $A$, it turns out that in the steady state the relative holding of capital stock will increase (decrease), that is the distribution of capital will become more unequal (equal) if $k_{0, i} / z_{0, i}<(>) k_{0}^{a} / z_{0}^{a}$. Again this is due to the different effect of the increase in $A$ on the saving rates. In fact, the initial saving rates ratio $s_{0}^{R}$ increases, following an increase in $A$, if $k_{0, i} / z_{0, i}<k_{0}^{a} / z_{0}^{a} .{ }^{16}$

As in the model with intertemporally dependent preferences just described, an evolving distribution of capital holdings is possible, even if with some limitations, in the $A k$ model with Stone-Geary preferences:

$$
u\left(c_{i}\right)=\frac{\left(c_{i}-\bar{c}\right)^{1-\sigma}}{1-\sigma}, \quad \sigma>0, \neq 1
$$

\footnotetext{
${ }^{16}$ If one wants to consider the effects of an increase in steady state growth on the evolution of inequality along the whole path, the relationship might be non-monotonic and will depend on how far the economy is from the balanced growth path.
} 
where the positive constant $\bar{c}$ is the subsistence level of consumption. This specification of preferences, used in a growth setting by Christiano (1989), Rebelo (1992) and Chatterjee and Ravikumar (1999).

It is easy to show that, with the Stone-Geary utility function, in equilibrium:

$$
k_{t, i}=\bar{k}+\left(k_{0, i}-\bar{k}\right) e^{g t},
$$

where $k_{0, i}>\bar{k}, \quad \bar{k} \equiv(\bar{c} / A)$, and the positive constant $\bar{c}$ is the subsistence level of consumption. Since an $A k$ technology is assumed, $\bar{k}$ can be interpreted as the amount of capital needed to produce the subsistence level of consumption. Furthermore,

$$
\begin{gathered}
g_{y, i}=\left(k_{t, i}\right)^{-1} g\left(k_{0, i}-\bar{k}\right) e^{g t}>0, \\
\dot{g}_{y, i}=\left(k_{t, i}\right)^{-2} g^{2}\left(k_{0, i}-\bar{k}\right) e^{g t} \bar{k}>0,
\end{gathered}
$$

where $g=\frac{A-\delta}{\sigma}$. These are the same solutions that would be obtained assuming the functional form (23) and setting $\rho=0$ and $z_{0, i} \equiv \bar{c} \forall i$, so that $z_{t, i}=z_{0, i} \equiv \bar{c} \forall t \forall i$, and the customary consumption is just constant at the subsistence level $\bar{c}$, which is the same for all the individuals. Comparing (24) and (27), it is clear that - because it allows, for each agent, a changing level of $z-(23)$ is generally consistent with a wider range of possibilities in terms of transitional dynamics toward the steady state growth path: while $\rho=0$ (and therefore a Stone-Geary utility function) yields the implication that the growth rate of per-capita output and the saving rate must necessarily be increasing over time along the transition, they can be either increasing or decreasing when $\rho \neq 0$ and the dynamics of habits feed back to consumption and accumulation choices.

Moreover, it can be shown that a Stone-Geary utility function implies that inequality always increases along the transition (see Chatterjee and Ravikumar, 1999) and, finally, that a positive shock to $A$ makes the steady state distribution of capital more concentrated. In fact, given this utility function, in equilibrium:

$$
k_{t, i}=\bar{k}+\left(k_{0, i}-\bar{k}\right) e^{g t},
$$

where $k_{0, i}>\bar{k}$. It follows that the steady state ratio of individual $i$ 's capital to the mean is given by

$$
\left(\bar{k}^{R}\right)^{*}=\frac{\left(A k_{0, i}-\bar{c}\right)}{\left(A k_{0}^{a}-\bar{c}\right)},
$$

which is decreasing in $A$ for $k_{0, i}>k_{0}^{a}$ and increasing for $k_{0, i}<k_{0}^{a}$. 


\section{Concluding remarks}

In this paper, we have provided a full characterization of the dynamics of the economy under internal habit formation and a linear technology. Rather than assuming a specific functional form for the instantaneous utility function, we have derived the restrictions that have to be placed on this latter to guarantee the existence of a balanced growth path, and introduced a simple graphical apparatus that greatly simplifies the study of the transitional dynamics starting from an arbitrary set of initial conditions on the state variables. We have also explored the implications of the model for the undertstanding of the evolution of the cross-section of wealth, concluding that they are not inconsistent with the, still scarce, empirical evidence.

We think that the main contribution of the present paper lies in the analysis of the rich dynamics stemming from a plausible, and tractable, departure from the assumption of time-separable, isoelastic preferences. These dynamics should be superimposed on those implied by models that give a more realistic account of the production side of the economy, allow for the existence of barriers to the international diffusion and adoption of technology, take into account the role played by Governments and institutions and, more generally, the countless factors we have deliberately neglected, but that which undoubtedly play an important role in the process of growth.

\section{References}

[1] Abel, A. B. (1990), "Asset Prices Under Habit Formation and Catching Up with the Joneses", American Economic Review Papers and Proceedings, vol. 80: pp. 38-42.

[2] Aghion, P., E. Caroli and C. Garcia-Penalosa (1999) "Inequality and Economic Growth: the Perspective of the New Growth Theories", Journal of Economic Literature, vol. 37: pp. 1615-1660.

[3] Becker, G. S. and K. M. Murphy (1988), "A Theory of Rational Addiction", Journal of Political Economy, vol. 96: pp. 675-700.

[4] Benabou, R. (1996) "Inequality and growth", in B.S. Bernanke and J.J. Rotemberg (eds.), NBER Macroeconomics Annual, pp. 1174. Cambridge, MA: MIT Press. 
[5] Boldrin, M., L. Christiano and J. Fisher (1997), "Habit Persistence and Asset Returns in an Exchange Economy", Macroeconomic Dynamics, vol. 1: pp. 312-332.

[6] Boyer, M. (1975), "An Optimal Growth Model with Stationary NonAdditive Utilities", Canadian Journal of Economics, vol. 8: pp. 216237.

[7] — (1978), "A Habit Forming Optimal Growth Model", International Economic Review, vol. 19: pp. 585-609.

[8] Caballé, J. and M.S. Santos (1993), "On Endogenous Growth with Physical and Human Capital", Journal of Political Economy, vol. 101, n. 6: pp. 1042-1067.

[9] Campbell, J. and J. Cochrane (1999), "By Force of Habit: A Consumption-Based Explanation of Aggregate Stock Market Behavior", Journal of Political Economy, vol. 107 n. 2: pp. 205-251.

[10] Carroll, C. and M. Kimball (1996), "On the Concavity of the Consumption Function", Econometrica, vol. 64 no. 2: pp.981-992.

[11] Carroll, C., J. Overland and D. Weil (1997), "Comparison Utility in a Growth Model", Journal of Economic Growth, vol. 2 n. 4: pp.339-367.

[12] — (2000) "Saving and Growth with Habit Formation", American Economic Review, vol. 90: pp. 341-355.

[13] Caselli, F. and J. Ventura (2000), "A Representative Consumer Theory of Distribution", American Economic Review, vol. 90: pp. 909-926.

[14] Chatterjee and Ravikumar (1999), "Minimum Consumption Requirements: Theoretical and Quantitative Implications for Growth and Redistribution", Macroeconomic Dynamics, vol. 3: pp.482-505.

[15] Constantinides, G. (1990). "Habit Formation: A Resolution of the Equity Premium Puzzle", Journal of Political Economy, vol. 98: pp. 519543.

[16] Deaton, A.S. (1992), "Understanding Consumption", Oxford University Press, New York.

[17] Deininger, K., and L. Squire (1998) “ A New Data Set Measuring Income Inequality", World Bank Economic Review, vol. 10: pp. 565-591. 
[18] Diaz, A., J. Pijoian-Mas and J.V. Rios-Rull (forthcoming) "Precautionary Savings and Wealth Distribution under Habit Formation Preferences", Journal of Monetary Economics.

[19] Fuhrer, C.J. and M.W. Klein (1998), "Risky Habits: On Risk Sharing, Habit Formation, and the Interpretation of International Consumption Correlations", NBER Working Paper n. 6735.

[20] Gali, J. (1994), "Keeping Up with the Joneses: Consumption Externalities, Portfolio Choice, and Asset Prices", Journal of Money, Credit, and Banking, vol. 26, no. 1: pp. 1-8.

[21] Heckman, J.J. (1976), "A Life-Cycle Model of Earnings, Learning, and Consumption", Journal of Political Economy, vol. 84, no. 4, Part 2: pp. S11-S44.

[22] Lattau, M. and H. Uhlig (2000), "Can Habit Formation Be Reconciled with Business Cycle Facts?", Revew of Economic Dynamics, Vol. 3, No. 1: pp. 79-99.

[23] Ljungqvist, L. and H. Uhlig. (2000), "Tax Policy and Agregate Demand Management Under Catching Up with the Joneses", American Economic Review, vol. 90, No.3: pp. 356-366.

[24] Mansoorian, A. (1993), "Habit Persistence and the Harberger-LaursenMetzler Effect in an Infinite Horizon Model", Journal of International Economics, vol. 34: pp. 153-166.

[25] Obstfeld, M. (1992), "International Adjustment with Habit-Forming Consumption: A Diagrammatic Exposition", Review of International Economics, vol. 1 n. 1: pp. 32-48.

[26] Otrok, C. (2001), "On measuring the Welfare Cost of Business Cycles", Journal of Monetary Economics, vol. 47: pp. 61-92.

[27] Rebelo, S. (1991), "Long-Run Policy Analysis and Long-Run Growth", Journal of Political Economy, vol. 99, n. 3: pp. 500-521.

[28] Ryder, H. E. Jr. and G. M. Heal (1973), "Optimal Growth with Intertemporally Dependent Preferences", Review of Economic Studies, vol. 40: pp. 1-33. 


\section{Appendix 1 - Proof of Proposition 1}

The current-value Hamiltonian for problem (P1) is:

$$
H=u(c, z)+\lambda(A k-c)+\mu \rho(c-z),
$$

where $\lambda$ and $\mu$ are the co-state variables associated with $k$ and $z$, respectively.

It follows that, among the necessary conditions for (P1), we have:

$$
\begin{aligned}
u_{c}+\rho \mu & =\lambda, \\
\dot{\lambda} & =(\delta-A) \lambda, \\
\dot{\mu} & =(\rho+\delta) \mu-u_{z}
\end{aligned}
$$

Differentiating (A.1.1) with respect to time, and using (A.1.2)-(A.1.3), one gets:

$$
u_{c c} \dot{c}+u_{c z} \dot{z}=(\delta+\rho) u_{c}-(A+\rho) \lambda+\rho u_{z},
$$

that, rearranging, can be written as follows:

$$
\frac{c u_{c c}(\dot{c} / c)+z u_{c z}(\dot{z} / z)}{u_{c}}=(\delta+\rho)-(A+\rho) \frac{\lambda}{u_{c}}+\rho \frac{u_{z}}{u_{c}} .
$$

To be an equilibrium, a balanced growth path must satisfy (A.1.4). Since $c$ and $z$ grow at the common rate $g$ in balanced growth, in steadystate equilibrium (A.1.4) becomes:

$$
g\left[\frac{c u_{c c}+z u_{c z}}{u_{c}}\right]=(\delta+\rho)-(A+\rho) \frac{\lambda}{u_{c}}+\rho \frac{u_{z}}{u_{c}} .
$$

Let's assume that $u$ is homogeneous of degree $\nu$ in $(c, z)$, so that $u_{c}$ is homogeneous of degree $(\nu-1)$ in the same variables. Euler's theorem then implies:

$$
\left[\frac{c u_{c c}+z u_{c z}}{u_{c}}\right]=\nu-1
$$

so that the left-hand side of (A.1.5) is a constant. For a balanced growth path to be an equilibrium, the right-hand side of (A.1.5) must be constant as well. Since homogeneity of $u$ implies that the term $\left(u_{c} / u_{z}\right)$ is a function of the ratio $(c / z)$ only - a constant in steady state growth - , this requires:

$$
\frac{\dot{u}_{c}}{u_{c}}=\frac{\dot{\lambda}}{\lambda}
$$


or, using (A.1.2) and evaluating at the steady state the rate of change of the marginal utility of consumption,

$$
g(\nu-1)=\delta-A .
$$

Rearranging, one obtains $\nu=\frac{\delta-A}{g}+1$, or the equivalent expression for the relationship between the degree of homogeneity of $u$ and $g$ given by equation (6) in the text. Notice that the requirement of positive steady state growth and Assumption T1 imply $\nu<1$.

Finally, it is easy to verify that, in steady state growth, $(\dot{\mu} / \mu)=$ $(\dot{\lambda} / \lambda)=\delta-A$. It follows that, for a balanced growth path to satisfy the transversality conditions associated with problem (P1),

$$
\begin{aligned}
& \lim _{t \rightarrow \infty} e^{-\delta t} \lambda_{t} k_{t}=0, \\
& \lim _{t \rightarrow \infty} e^{-\delta t} \mu_{t} z_{t}=0,
\end{aligned}
$$

one must have $A>g$. We restrict the parameters in the model so as to make sure that this inequality always holds, implying that the balanced growth path just characterized satisfies all the necessary - and, given our assumptions, sufficient - conditions for an optimum.

\section{Appendix 2 - Derivation of equations (17a)-(17c)}

Since $\tilde{k}$ does not enter (14a)- (14b), we begin our local analysis of the equilibrium dynamics associated with system (14) by focusing on the pair $(\tilde{c}, \tilde{z})$. The dynamics of $\tilde{k}$ follows recursively, through (14c).

Linearizing (14a)-(14b) around the steady state, one gets:

$$
\left[\begin{array}{c}
\dot{\tilde{c}} \\
\dot{\tilde{z}}
\end{array}\right]=J \cdot\left[\begin{array}{c}
\tilde{c}-\tilde{c}^{*} \\
\tilde{z}-\tilde{z}^{*}
\end{array}\right]
$$

where

$$
J \equiv\left[\begin{array}{cc}
A+\rho & -j \\
\rho & -(\rho+g)
\end{array}\right],
$$

and $j$ is given by (16) in the text. The two roots of the characteristic equation associated with system (A.2.1) are:

$$
\frac{(A-g)}{2} \pm \frac{\sqrt{\Delta}}{2}
$$


where

$$
\begin{aligned}
\Delta & \equiv(A-g)^{2}+4(A+\rho)(\rho+g)-4 \rho j \\
& =4 \cdot\left\{\left[\frac{(A+g)}{2}+\rho\right]^{2}-\rho j\right\} .
\end{aligned}
$$

Using the definition of $j$ :

$$
\left[\frac{(A+g)}{2}+\rho\right]^{2}-\rho j=\left(\frac{1}{u_{\tilde{c} \tilde{c}}^{*}}\right) \cdot\left\{\left[\frac{(A+g)}{2}+\rho\right]^{2} u_{\tilde{c} \tilde{c}}^{*}+2 \rho\left[\frac{(A+g)}{2}+\rho\right] u_{\tilde{c} \tilde{z}}^{*}+\rho^{2} u_{\tilde{z} \tilde{z}}^{*}\right\} \geq 0
$$

because the term in curly brackets is a quadratic form in $\left[\frac{(A+g)}{2}+\rho\right]$ and $\rho$, and the Hessian of $u$ is negative semidefinite.

It follows that concavity of the instantaneous felicity function implies:

$$
\rho j \leq\left[\frac{(A+g)}{2}+\rho\right]^{2},
$$

so that $\Delta \geq 0$, and the two roots in (A.2.2) are real.

Given this result, and the fact that $\operatorname{Trace}(J)=A-g>0$, their sign can be determined on the basis of the sign of the determinant:

$$
|J|=\rho j-(A+\rho)(\rho+g) .
$$

When $j<0$, the jacobian determinant is negative, and we have two real roots of opposite sign. To make sure that the system is saddlepath stable also in the case of adjacent complementarity $(j>0)$ on which we focus in the text, we assume:

$$
\rho j<(A+\rho)(\rho+g),
$$

which amounts to the restriction on $j$ in (16). Notice that, being $(A+\rho)(\rho+g)<\left[\frac{(A+g)}{2}+\rho\right]^{2}$, any value of $j$ consistent with saddlepath stability is also consistent with concavity of $u$, and the upper bound that this assumption imposes on $j$ (see (A.2.3)).

We shall denote by $\zeta$ the positive characteristic root associated with the linearized version of system (14), and, as stated in the text, by $-\psi$ the negative one:

$$
-\psi=\frac{(A-g)}{2}-\sqrt{\left[\frac{(A+g)}{2}+\rho\right]^{2}-\rho j} .
$$


Notice that, although $j$ depends on the second partial derivatives of $u$ evaluated at the steady state $\left(\tilde{c}^{*}, \tilde{z}^{*}\right)$, homogeneity of $u$ implies that these roots are not a function of the initial conditions $\left(k_{0}, z_{0}\right)$. In fact,

$$
\begin{aligned}
j & =-\frac{(A+g+2 \rho) u_{\tilde{c} \tilde{z}}\left(\tilde{c}^{*}, \tilde{z}^{*}\right)+\rho u_{\tilde{z} \tilde{z}}\left(\tilde{c}^{*}, \tilde{z}^{*}\right)}{u_{\tilde{c} \tilde{c}}\left(\tilde{c}^{*}, \tilde{z}^{*}\right)} \\
& =-\frac{(A+g+2 \rho)\left(\tilde{c}^{*}\right)^{\nu-2} u_{\tilde{c} \tilde{z}}\left(1, \frac{\rho}{\rho+g}\right)+\rho\left(\tilde{c}^{*}\right)^{\nu-2} u_{\tilde{z} \tilde{z}}\left(1, \frac{\rho}{\rho+g}\right)}{\left(\tilde{c}^{*}\right)^{\nu-2} u_{\tilde{c} \tilde{c}}\left(1, \frac{\rho}{\rho+g}\right)} \\
& =-\frac{(A+g+2 \rho) u_{\tilde{c} \tilde{z}}\left(1, \frac{\rho}{\rho+g}\right)+\rho u_{\tilde{z} \tilde{z}}\left(1, \frac{\rho}{\rho+g}\right)}{u_{\tilde{c} \tilde{c} \tilde{c}}\left(1, \frac{\rho}{\rho+g}\right)} .
\end{aligned}
$$

It follows that changes in the initial conditions will determine parallel upward or downward shifts of the saddlepaths in Figures 1 and 2.

For later use, let's define the two constants $\omega_{1} \equiv \frac{\rho}{\rho+g-\psi}, \omega_{2} \equiv \frac{1}{A-g+\psi}$. While the second one is always positive, the sign of $\omega_{1}$ depends on that of $(\rho+g-\psi)$, which is the same as the sign of $j$. To see this, notice that , using (A.2.4):

$$
\rho+g-\psi=\left[\frac{(A+g)}{2}+\rho\right]-\sqrt{\left[\frac{(A+g)}{2}+\rho\right]^{2}-\rho j},
$$

where we know that the term under the radical is positive. It follows that $(\rho+g-\psi) \gtrless 0$ as $j \gtrless 0$.

These definitions and results imply that the general solution of system (A.2.1) can be written as follows:

$$
\begin{aligned}
& \tilde{c}_{t}-\tilde{c}^{*}=\Omega_{1} e^{-\psi t}+\Omega_{2} e^{\zeta t}, \\
& \tilde{z}_{t}-\tilde{z}^{*}=\omega_{1} \Omega_{1} e^{-\psi t}+\left(\frac{\rho}{\rho+g+\zeta}\right) \Omega_{2} e^{\zeta t},
\end{aligned}
$$

where $\Omega_{1}$ and $\Omega_{2}$ are arbitrary constants, to be determined using the initial conditions on the state variables and the transversality conditions in (12). Using (A.2.5) in the linearized version of the law of motion of $\tilde{k}$ and solving the resulting first-order, non-autonomous differential equation, yields:

$$
\begin{gathered}
\tilde{k}_{t}-\tilde{k}^{*}=\left[\left(k_{0}-\tilde{k}^{*}\right)-\omega_{2} \Omega_{1}\right] e^{(A-g) t}+\omega_{2} \Omega_{1} e^{-\psi t}+ \\
\left(\frac{\Omega_{2}}{g-A+\zeta}\right)\left(e^{(A-g) t}-e^{\zeta t}\right) .
\end{gathered}
$$


Being $\tilde{\lambda}$ constant, the first transversality condition in (12) requires:

$$
\begin{aligned}
& \lim _{t \rightarrow \infty} e^{-(A-g) t} \tilde{k}_{t}=\lim _{t \rightarrow \infty}\left\{e^{-(A-g) t} \tilde{k}^{*}+\left[\left(k_{0}-\tilde{k}^{*}\right)-\omega_{2} \Omega_{1}\right]+\right. \\
&\left.\omega_{2} \Omega_{1} e^{-(A-g+\psi) t}+\left(\frac{\Omega_{2}}{g-A+\zeta}\right)\left(1-e^{[\zeta-(A-g)] t}\right)\right\}=0
\end{aligned}
$$

Since $\zeta-(A-g)=\psi>0$, for the transversality condition on the capital stock to be met one must have:

$$
\begin{aligned}
& \Omega_{2}=0, \\
& \Omega_{1}=\left(\frac{1}{\omega_{2}}\right)\left(k_{0}-\tilde{k}^{*}\right),
\end{aligned}
$$

which imply that, in equilibrium, (A.2.7) becomes:

$$
\tilde{k}_{t}-\tilde{k}^{*}=\left(k_{0}-\tilde{k}^{*}\right) e^{-\psi t} .
$$

Now notice that, using (A.2.8) in (A.2.5)-(A.2.6), evaluating at time $t=0$ the resulting expressions for $\left(\tilde{c}_{t}-\tilde{c}^{*}\right)$ and $\left(\tilde{z}_{t}-\tilde{z}^{*}\right)$, and taking (A.2.9) into account, one obtains:

$$
\tilde{c}_{0}-\tilde{c}^{*}=\left(\frac{1}{\omega_{1}}\right)\left(z_{0}-\tilde{z}^{*}\right)=\left(\frac{1}{\omega_{2}}\right)\left(k_{0}-\tilde{k}^{*}\right) .
$$

Plugging the expressions for $\tilde{z}^{*}$ and $\tilde{k}^{*}$ as a function of $\tilde{c}^{*}$ given by (15a)(15b) into the second of these equalities, one gets:

$$
\tilde{c}^{*}=\frac{(A-g)(\rho+g)}{\psi(A+\rho)}\left[-\frac{1}{\omega_{1}} z_{0}+\frac{1}{\omega_{2}} k_{0}\right]
$$

this result, used in the first equality in (A.2.11), yields:

$$
\tilde{c}_{0}=\frac{(\rho+g)}{(A+\rho) \omega_{1}} z_{0}+\frac{(A-g)}{(A+\rho) \omega_{2}} k_{0} .
$$

Finally, it is immediate to verify that (A.2.5), (A.2.6) and (A.2.10) can be written as (17a)-(17c) in the text, with $\tilde{c}_{0}$ and $\tilde{c}^{*}$ taking on the values just derived. Since these equilibrium paths imply convergence to a steady state in which all variables assume constant values, they also satisfy the second transversality condition in (12). 


\section{Appendix 3 - Global dynamics in the subtractive formulation of preferences}

In this Appendix we prove the claim made in the text that, when the instantaneous utility function is (23), the equilibrium paths (17a)-(17c) derived by linearizing system (14) around the steady state (15) also describe the global - and not just the local - equilibrium dynamics of $\left(\tilde{c}_{i}, \tilde{z}_{i}, \tilde{k}_{i}\right)$, and therefore $\left(c_{i}, z_{i}, k_{i}\right)$.

First notice that, with $u$ given by (24), we have: $u_{c}=\left(c_{i}-z_{i}\right)^{-\sigma}$; $u_{z}=-\left(c_{i}-z_{i}\right)^{-\sigma} ; u_{c c}=-\sigma\left(c_{i}-z_{i}\right)^{-\sigma-1} ; u_{z z}=-\sigma\left(c_{i}-z_{i}\right)^{-\sigma-1} ; u_{c z}=$ $\sigma\left(c_{i}-z_{i}\right)^{-\sigma-1}$. It follows that (14a)-(14b) can be equivalently written as:

$$
\begin{aligned}
\dot{\tilde{c}}_{i}-\dot{\tilde{z}}_{i} & =B\left(\tilde{c}_{i}-\tilde{z}_{i}\right)^{\sigma+1}-\left(\frac{A}{\sigma}\right)\left(\tilde{c}_{i}-\tilde{z}_{i}\right), \\
\dot{\tilde{z}}_{i} & =\rho \tilde{c}_{i}-(\rho+g) \tilde{z}_{i},
\end{aligned}
$$

where $B \equiv \frac{\tilde{\lambda}^{*}(A+\rho)}{\sigma}>0$. Next, define $\eta_{i} \equiv \tilde{c}_{i}-\tilde{z}_{i}$, and rewrite the above system as follows:

$$
\begin{aligned}
& \dot{\eta}_{i}=B \eta_{i}^{\sigma+1}-\left(\frac{A}{\sigma}\right) \eta_{i}, \\
& \dot{\tilde{z}}_{i}=\rho \eta_{i}-g \tilde{z}_{i} .
\end{aligned}
$$

The phase diagram for this system is shown in Fig. A3. The only initial choice of $\eta_{i}$ consistent with convergence to the steady state $\eta_{i}^{*}=\left(\frac{A}{\sigma B}\right)^{1 / \sigma}$, $\tilde{z}_{i}^{*}=\frac{\rho}{g}\left(\frac{A}{\sigma B}\right)^{1 / \sigma}$ is $\eta_{0, i}=\eta_{i}^{*}$, so that $\eta_{t, i}=\eta_{0, i}=\left(\frac{A}{\sigma B}\right)^{1 / \sigma} \forall t$. Other choices of $\eta_{0, i}$ would lead either to a situation in which $\eta_{i}$ hits zero in finite time (which cannot be optimal, being $\lim _{\eta_{i} \rightarrow 0} u_{c}=\infty$ ), or to a violation of the transversality condition on $\tilde{z}_{i}$.

Plugging $\eta_{t, i}=\eta_{0, i}$ into the law of motion of $\tilde{z}_{i}$, and solving the resulting autonomous differential equation, yields:

$$
\tilde{z}_{t, i}=\left[z_{0, i}-\left(\frac{\rho}{g}\right) \eta_{0, i}\right] e^{-g t}+\left(\frac{\rho}{g}\right) \eta_{0, i}
$$

so that:

$$
\tilde{c}_{t, i}=\tilde{z}_{t, i}+\eta_{0, i}=\left[z_{0, i}-\left(\frac{\rho}{g}\right) \eta_{0, i}\right] e^{-g t}+\left(\frac{\rho+g}{g}\right) \eta_{0, i} .
$$


Using this expression for $\tilde{c}_{t, i}$ into the law of motion of $\tilde{k}_{i}$, and integrating, one gets:

$$
\tilde{k}_{t, i}=k_{0, i} e^{(A-g) t}+\left[\frac{\rho+g}{g(A-g)}\right] \eta_{0, i}\left(1-e^{-(A-g) t}\right)+\left(\frac{1}{A}\right)\left[z_{0, i}-\left(\frac{\rho}{g}\right) \eta_{0, i}\right]\left(e^{-g t}-e^{(A-g) t}\right) .
$$

Since the transversality condition $\lim _{t \rightarrow \infty} e^{-(A-g) t} \tilde{k}_{t, i}=0$ requires:

$$
\eta_{0, i}=\left(\frac{A-g}{A+\rho}\right)\left(A k_{0, i}-z_{0, i}\right)
$$

in equilibrium:

$$
\tilde{k}_{t, i}=\left[\frac{\rho+g}{g(A+\rho)}\right]\left(A k_{0, i}-z_{0, i}\right)+\left[\frac{\rho+g}{g(A+\rho)}\right]\left[z_{0, i}-\left(\frac{\rho(A-g)}{\rho+g}\right) k_{0, i}\right] e^{-g t} .
$$

Finally, using (A.3.3) into (A.3.1) and (A.3.2) yields:

$$
\begin{aligned}
& \tilde{z}_{t, i}=\left[\frac{\rho(A-g)}{g(A+\rho)}\right]\left(A k_{0, i}-z_{0, i}\right)+\left[\frac{A(\rho+g)}{g(A+\rho)}\right]\left[z_{0, i}-\left(\frac{\rho(A-g)}{\rho+g}\right) k_{0, i}\right] e^{-g t}, \\
& \tilde{c}_{t, i}=\left[\frac{(A-g)(\rho+g)}{g(A+\rho)}\right]\left(A k_{0, i}-z_{0, i}\right)+\left[\frac{A(\rho+g)}{g(A+\rho)}\right]\left[z_{0, i}-\left(\frac{\rho(A-g)}{\rho+g}\right) k_{0, i}\right] e^{-g t} .
\end{aligned}
$$

These are the same equilibrium paths one would get using (17)-(19) and setting $\omega_{1}=1, \omega_{2}=(1 / A), \quad \psi=g$, the values these constants take on when the instantaneous utility function is (23). 
Figure 1

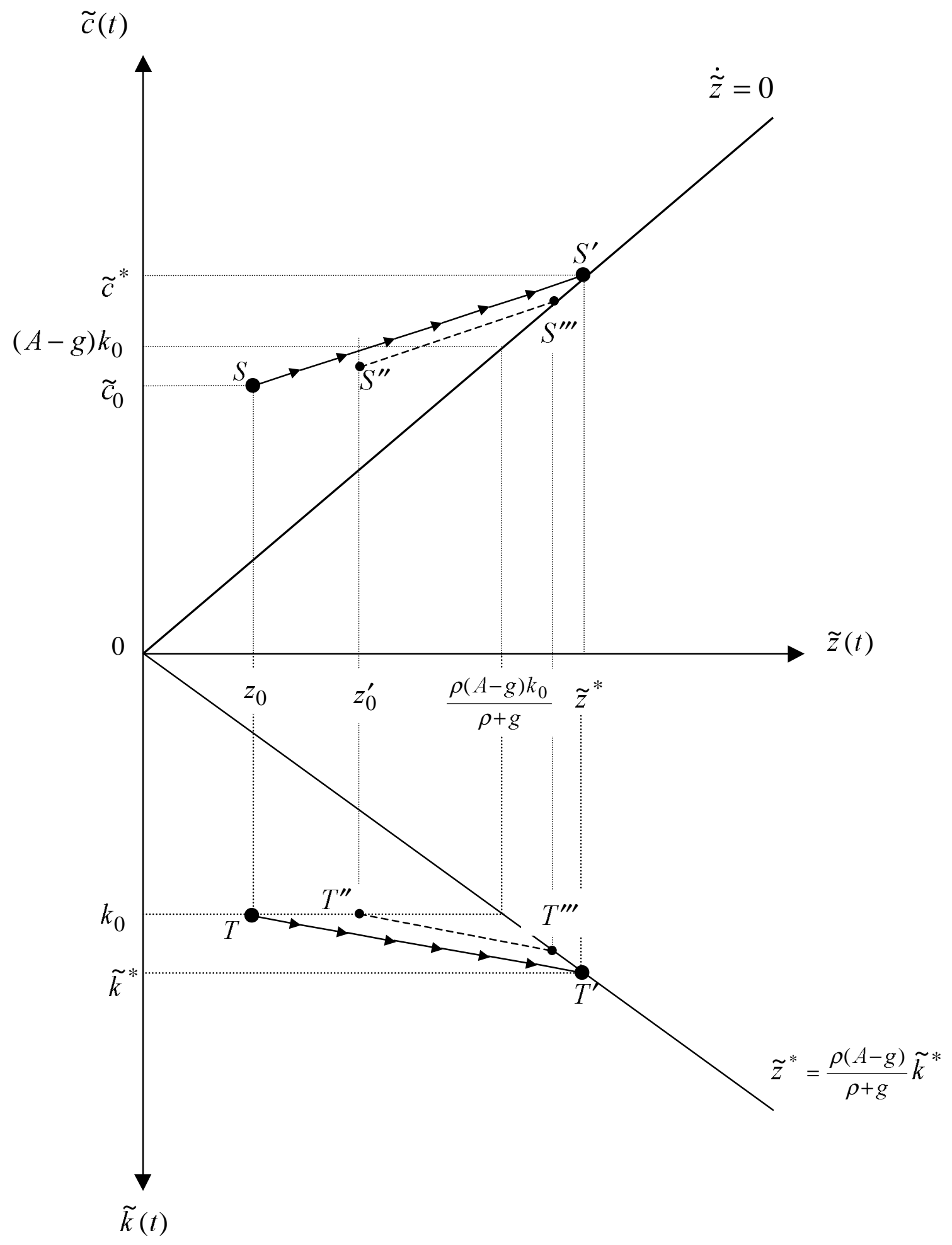


Figure 2

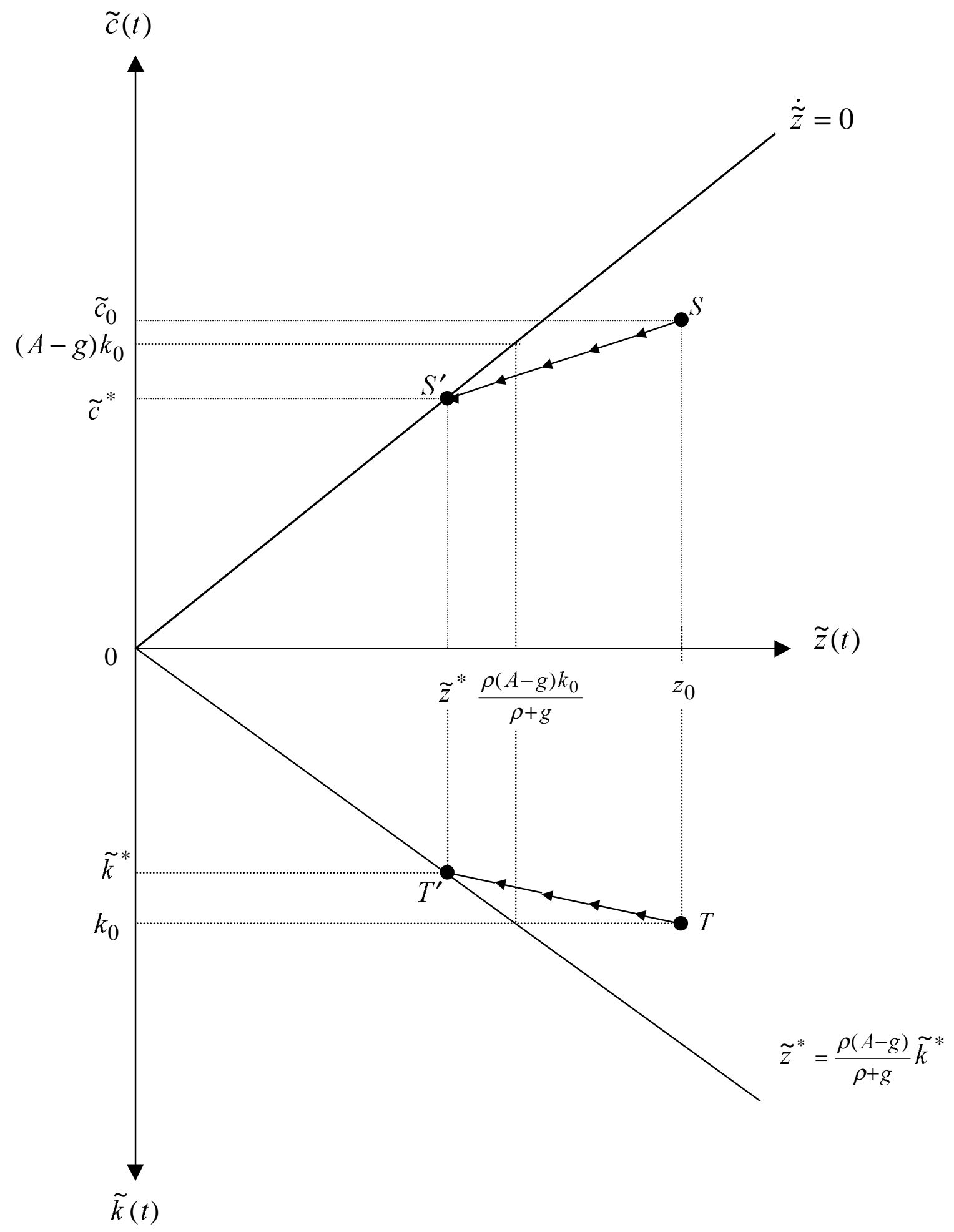

